\title{
Clinically accessible neuroimaging predictors of post-stroke neurocognitive disorder: a prospective observational study
}

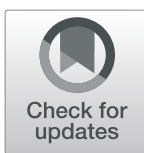

Till Schellhorn ${ }^{1,2^{*}}$ D, Eva Birgitte Aamodt ${ }^{1,2}$, Stian Lydersen ${ }^{3}$, Stina Aam ${ }^{4,5}$, Torgeir Bruun Wyller ${ }^{2,6}$, Ingvild Saltvedt ${ }^{4,5}$ and Mona Kristiansen Beyer ${ }^{1,2}$

\begin{abstract}
Background: Neurocognitive disorder (NCD) is common in stroke survivors. We aimed to identify clinically accessible imaging markers of stroke and chronic pathology that are associated with early post-stroke NCD.

Methods: We included 231 stroke survivors from the "Norwegian Cognitive Impairment after Stroke (Nor-COAST)" study who underwent a standardized cognitive assessment 3 months after the stroke. Any NCD (mild cognitive impairment and dementia) and major NCD (dementia) were diagnosed according to "Diagnostic and Statistical Manual of Mental Disorders (DSM-5)" criteria. Clinically accessible imaging findings were analyzed on study-specific brain MRIs in the early phase after stroke. Stroke lesion volumes were semi automatically quantified and strategic stroke locations were determined by an atlas based coregistration. White matter hyperintensities (WMH) and medial temporal lobe atrophy (MTA) were visually scored. Logistic regression was used to identify neuroimaging findings associated with major NCD and any NCD.
\end{abstract}

Results: Mean age was 71.8 years (SD 11.1), 101 (43.7\%) were females, mean time from stroke to imaging was 8 (SD 16) days. At 3 months 63 (27.3\%) had mild NCD and 65 (28.1\%) had major NCD. Any NCD was significantly associated with WMH pathology (odds ratio $(\mathrm{OR})=2.73$ [1.56 to 4.77], $p=0.001$ ), MTA pathology (OR=1.95 [1.12 to 3.41], $p=0.019$ ), and left hemispheric stroke ( $\mathrm{OR}=1.8$ [1.05 to 3.09], $p=0.032$ ). Major NCD was significantly associated with $\mathrm{WMH}$ pathology $(\mathrm{OR}=2.54$ [1.33 to 4.84], $p=0.005)$ and stroke lesion volume $(\mathrm{OR}(\mathrm{per} \mathrm{ml})=1.04$ [1.01 to 1.06], $p=0.001$ ).

Conclusion: WMH pathology, MTA pathology and left hemispheric stroke were associated with the development of any NCD. Stroke lesion volume and WMH pathology were associated with the development of major NCD 3 months after stroke. These imaging findings may be used in the routine clinical setting to identify patients at risk for early post-stroke NCD.

Trial registration: ClinicalTrials.gov, NCT02650531, Registered 8 January 2016 - Retrospectively registered.

Keywords: Stroke imaging, Cognitive impairment, Post stroke dementia, Stroke volume, White matter lesions

\footnotetext{
* Correspondence: tillsc@uio.no

'Division of Radiology and Nuclear Medicine, Oslo University Hospital, Oslo, Norway

${ }^{2}$ Institute of Clinical Medicine, University of Oslo, Oslo, Norway

Full list of author information is available at the end of the article
}

(C) The Author(s). 2021 Open Access This article is licensed under a Creative Commons Attribution 4.0 International License, which permits use, sharing, adaptation, distribution and reproduction in any medium or format, as long as you give appropriate credit to the original author(s) and the source, provide a link to the Creative Commons licence, and indicate if changes were made. The images or other third party material in this article are included in the article's Creative Commons licence, unless indicated otherwise in a credit line to the material. If material is not included in the article's Creative Commons licence and your intended use is not permitted by statutory regulation or exceeds the permitted use, you will need to obtain permission directly from the copyright holder. To view a copy of this licence, visit http://creativecommons.org/licenses/by/4.0/. The Creative Commons Public Domain Dedication waiver (http://creativecommons.org/publicdomain/zero/1.0/) applies to the data made available in this article, unless otherwise stated in a credit line to the data. 


\section{Background}

Neurocognitive disorder (NCD), which includes mild and major NCD, is common after stroke $[1,2]$ and a major cause of disability for stroke survivors [3, 4]. Mild and major NCD are the latest nomenclature for the previously used diagnoses "mild cognitive impairment" and "dementia", defined in the most recent "Diagnostic and Statistical Manual of Mental Disorders (DSM-5)" [5, 6]. Reported prevalence of major NCD after a first-ever stroke ranges from 10 to $40 \%$ [2], and is stated to be around $30 \%$ for recurrent stroke [7]. Up to $71 \%$ of stroke patients are reported to have mild NCD 3 months post stroke, depending on assessment methods and diagnostic criteria used [1]. Three months after stroke, the prevalence in the "Norwegian Cognitive Impairment after Stroke (Nor-COAST)" study were found to be 29 and $26 \%$ for mild and major NCD, respectively [8]. Age, education, stroke severity, and previous stroke seem to be clinically important factors for the development of NCD, while white matter hyperintensities (WMH), medial temporal lobe atrophy (MTA) [9, 10], strategic stroke location, and total brain volume have been suggested as important imaging findings associated with the development of cognitive impairment [11, 12]. Seminal neuropathological observations by Tomlinson et al., published in 1970 [13] and backed up by a recent study [14], suggested a relationship between stroke volume, stroke location, and cognitive impairment, while another recent study found WMH-volume and not the stroke volume to be a significant predictor of cognitive impairment after a mild stroke [15]. While WMHs are consistently stated as strong predictors of post-stroke cognitive impairment, the importance of the stroke lesion volume is still disputable [16], especially for small stroke volumes $(\sim 5 \mathrm{ml})$ that are commonly observed in the clinical routine [17].

In order to be clinically feasible, neuroimaging markers should be accessible on routine acute stroke imaging, not only on advanced research sequences. Fast routine stroke imaging combined with visual rating scales would allow for early clinical predictions of poststroke NCD without the need for complicated MRI post-processing software and advanced sequences. The clinical translation of this study is the identification of robust robust neuroimaging markers of post-stroke NCD, which will help to estimate the risk of post-stroke cognitive impairment for the individual patients and allow the identification of patients susceptible to preventive interventions.

The aim of this study was to identify clinically accessible neuroimaging markers that are associated with early post-stroke NCD.

Our hypothesis was that patients with larger stroke volume, left-sided stroke, strategic stroke locations, higher WMH burden and higher MTA-score more frequently develop post-stroke NCD.

\section{Methods \\ Nor-COAST}

The current study uses data from the Norwegian Cognitive Impairment After Stroke (Nor-COAST) study; a prospective longitudinal multicenter cohort study recruiting patients hospitalized with acute stroke in five Norwegian stroke units from May 2015 to March 2017. Details of the Nor-COAST study are described by Thingstad et al. [18].

\section{Population}

All patients admitted to the five stroke units participating in Nor-COAST with suspected stroke were screened for inclusion. A stroke was diagnosed according to the World Health Organisation (WHO) criteria [19] as a focal (or at times global) neurological impairment of sudden onset, and lasting more than $24 \mathrm{~h}$ (or leading to death) and of presumed vascular origin or an findings of acute infarction or intra-cerebral hemorrhage on MRI.

Eligible patients for the inclusion in Nor-COAST were according to the inclusion criteria (a) admitted with acute ischemic or hemorrhagic stroke hospitalized within 1 week after onset of symptoms; (b) age over 18 years; and (c) fluent in a Scandinavian language. Exclusion criteria were (a) symptoms explained by other disorders than ischemic brain infarct or intracerebral hemorrhages; (b) expected survival less than 3 months after stroke based on a clinical assessment by experienced stroke physicians. Patients with pre-existing cognitive impairment or dementia were not excluded. Inclusion criteria for the MRI substudy were (a) patient included in Nor-COAST; (b) modified Rankin scale $<5$ before the stroke; (c) able to cooperate during MRI. Exclusion criteria for MRI were (a) severe functional impairment making MRI impossible to perform; (b) medical contraindications for MRI like claustrophobia or pacemaker; (c) patient declined participation in MRI substudy. Participation in the MRI substudy was optional.

\section{MRI acquisition}

A study-specific brain MRI was performed as early as possible during the acute/subacute phase of the stroke. Brain scans were acquired at all five participating hospitals, using one specific MRI-scanner on each site (GE Discovery MR750, $3 \mathrm{~T}$; Siemens Biograph_mMR, $3 \mathrm{~T}$; Philips Achieva dStream, 1.5 T; Philips Achieva, 1.5 T; Siemens Prisma, $3 \mathrm{~T}$ ). The study protocol consisted of 3D-T1 weighted, axial T2, 3D-Fluid attenuated inversion recovery (FLAIR), diffusion-weighted imaging (DWI), 
and susceptibility-weighted imaging (SWI). Details about the MRI protocol have previously been described [20].

\section{Image analysis \\ Coregistration and stroke segmentation}

The 3D-T1 sequence was linearly registered to the $1 \mathrm{~mm}$ "Montreal Neurological Institue-152" (MNI-152) T1 template with the help of "Oxford Centre for Functional Magnetic Resonance of the Brain (FMRIB)" version 6.0 [21] Linear Image Registration Tool (FLIRT) [21, 22]. DWI trace images were coregistered and resampled to the already co-registered 3D-T1 using the same software (FLIRT).

The stroke lesion volume was defined as equivalent to the ischemic core that represents the amount of irreversibly destroyed brain parenchyma, identified as diffusion restriction on the DWI sequence. Acute infarcts were semi-automatically labeled with the help of the "Insight Segmentation and Registration Toolkit-Snap (ITKSnap)" snake tool (v. 3.8.0) [23] in order to create lesion masks. Hemorrhagic stroke lesions were included in this analysis. We did not split the analysis of stroke lesion volume in ischemic and hemorrhagic stroke because of the small number and comparable volume of hemorrhagic strokes. Lesion masks were created for all patients who had visible diffusion restriction on DWI. The masked stroke volume in $\mathrm{mm}^{3}$ was automatically measured by ITK-snap (v. 3.8.0), exported to a comma separated values (CSV) file, and converted to milliliters (ml).

\section{Localization of acute stroke}

Infarcts in the thalamus, angular gyrus, hippocampus, parahippocampal gyrus, caudate, substantia nigra, red nucleus, lateral and medial globus pallidus were considered strategic infarcts in accordance with previous findings [24, 25]. Coordinates of masked stroke lesions were used to identify the hemisphere and corresponding anatomical structure in Talairach brain atlases [26]. The anatomical atlas labels were retrieved with the help of the "AtlasReader" python package [27].

\section{Visually rated variables}

WMH of presumed vascular origin were classified according to the widely used Fazekas scale [28]. The acute stroke lesion was not included in the Fazekas score. Fazekas grade of 1 was considered normal for all ages. The following Fazekas grades were used to identify WMH pathology. Fazekas grade 2 was considered normal in patients 71 years or older, whereas Fazekas grade 3 was always regarded as pathological [29]. MTA was assessed according to the established MTA scale [30]. Findings were considered pathological where the mean MTA of both sides was $\geq 1.5$ under the age of 75 , a value $\geq 2$ below the age of 85 , and a value of $\geq 2.5$ below 95 years, as recommended by Ferreira et al. [31]. For a thorough description of the methods of visual assessment please see Schellhorn et al. [20]. Please see Supplement A4 for a description of the Fazekas scale, and MTA scale.

\section{Clinical characteristics}

Demographic and clinical variables were recorded at the time of the index stroke by study nurses and stroke physicians. The following data were included: Age, years of education, gender, stroke severity measured with the National Institute of Health Stroke Scale (NIHSS), hypertension, hypercholesterolemia, smoking, diabetes mellitus, clinical history of previous stroke and clinical stroke subtype according to the "Trial of ORG 10172 in Acute Stroke Treatment (TOAST)" classification [32].

\section{Cognitive assessment}

Pre-stroke cognitive impairment was assessed by interviews of relatives or caregivers, using the Global Deterioration Scale (GDS). The GDS ranges from 1 to 7. A GDS score of 3 or above is categorized as pathological [33], with 3 representing mild NCD and values from 4 to 7 are representing major NCD.

The diagnosis of post-stroke NCD was based on the "Diagnostic Statistical Manual of Mental Disorders (DSM5)" implementing neuropsychological test scores. Five of six cognitive domains cited in the DSM- 5 were assessed with a domain specific test: perceptual-motor function, language, executive function, learning and memory, and complex attention. Social cognition was not measured. The perceptual-motor function was tested with the visuospatial/executive domain from MoCA. Language was evaluated with the verbal fluency test category (animals). Executive function was measured with the trail making test B and verbal fluency test - letter (FAS). Learning and memory was evaluated with the word list recall test. Complex attention was measured with the trail making test - A [8].

Patients scoring $<-1.5$ SD in at least one cognitive domain were defined as having poststroke NCD. According to these criteria the cognitive status after 3 months was classified into normal cognition, mild NCD or major NCD. All patients with either mild or major NCD were classified as any NCD.

Major NCD was defined as post stroke NCD and dependency in I-ADL; whereas mild NCD was defined as post stroke NCD with independence in I-ADL, as previously published [8]. Moreover, cognitive status during hospital stay and after 3 months was additionally assessed with the "Montreal Cognitive Assessment Test (MoCA) [34], which is a comprehensive screening test used to detect NCD. 


\section{Statistical analysis}

In order to perform logistic regression analyses, the three cognitive outcome categories were dichotomized in two different ways: (a) normal cognition versus any NCD; and (b) major NCD versus normal cognition or mild NCD. This allowed us to separately analyze predictors of any NCD and major NCD. We attempted ordinal logistic regression with three possible cognitive outcomes (normal, mild NCD and major NCD) as the dependent variable. However, the ordinal logistic model turned out to be unsuited for our study as the odds ratios differed considerably between the thresholds.

The predictor variables stroke volume, strategic infarct, affected hemisphere, MTA pathology, and WMH pathology were analyzed one at a time for any NCD and major NCD using univariate logistic regression and all together in one multiple logistic regression model. We analyzed further if the pre-stroke cognitive status influenced the effect of the investigated predictor variables. For this purpose, the logistic regression analyses were repeated with pre-stroke GDS included in the logistic regression model.

All results were adjusted for the known important confounders age, sex, and education if not explicitly marked as unadjusted. Missing values were handled using available case analysis, that is, each analysis includes all the cases with available data on the variables in that analysis. Two-sided $p$-values $<0.05$ were considered as indicators of statistical significance, and we reported 95\% confidence intervals $(\mathrm{CI})$, where relevant. Statistical analyses were carried out using the software Stata 16 [35].

\section{Results}

\section{Study population}

The total Nor-COAST study population consisted of 815 participants. Study specific MRI scans that fulfilled all quality requirements were performed in 347 (42.6\%) of all Nor-COAST participants. The remaining 468 (57.4\%) were not eligible for this MRI study due to severe functional impairment, not able to cooperate, medical contraindication, incomplete or inadequate MRI-protocol, or because the patient declined participation in the MRI sub study. Of the 347 patients with study specific MRI scans 70 failed quality control due to technical reasons, movement or missing sequences. Cognitive status according to DSM- 5 criteria was performed in 231 (66.6\%) patients 3 months after the stroke, resulting in a study sample of 231. Participation in this study is shown in Fig. 1 below. The mean (SD) time to hospital admission was 13 [27] hours. The mean (SD) time to MRI was 8 [16] days.

For the whole study sample at baseline the mean age (SD) of patients was 71.8 (11.1), 101 (43.7\%) were female, mean length of education was 12.3 (3.7) years, $32(13.6 \%)$ had a history of previous clinical stroke. The mean (SD) MoCA score was 23.1 (3.1) 3 month after stroke. Stroke subtype according to TOAST classification $(n=208)$ : Large-vessel disease $18(8.7 \%)$, cardioembolic disease 44 (21.2\%), small-vessel disease 59 (28.4\%), other etiology 4 (1.9\%), undetermined etiology 83 (39.9\%). A hemorrhagic stroke was identified in 17 (7.4\%) patients. Mild pre-stroke NCD was evident in 13 (5.6\%) and major pre-stroke NCD in 10 (4.4\%) of the patients. A complete frequency table of GDS grades versus post-stroke cognitive outcome can be found in the Supplemental Table A1. Clinical characteristics grouped by cognitive outcome are summarized in Table 1.

\section{MRI}

\section{Imaging markers}

The mean (SD) stroke volume was $8.7(17.4) \mathrm{ml}$, the left hemisphere was affected in 102 (45.1\%) of the patients, both hemispheres in $6(2.7 \%), 23(9.9 \%)$ had suffered a strategic stroke. An overview of all affected locations is given in Supplemental Table A2. Pathologic WMH score was found in $90(39 \%)$ and $82(35.5 \%)$ had pathologic MTA score. Imaging findings grouped by cognitive outcome are summarized in Table 2. Imaging examples of WMH, MTA and stroke lesion volume for the three cognitive outcomes are given in Fig. 2.

\section{Cognitive outcome after 3 months}

Three months after the stroke 128 (55.4\%) had any NCD, mild NCD was found in $63(27.3 \%)$ and major NCD in 65 (28.1\%).

\section{Any NCD}

Any NCD after 3 months was significantly associated with WMH pathology (odds ratio $(\mathrm{OR})=2.73$ [1.56 to 4.77]), MTA pathology (OR $=1.95$ [1.12 to 3.41]) and left hemispheric stroke $(\mathrm{OR}=1.8 \quad[1.05$ to 3.09$])$. The resulting ORs remained approximately constant after adjusting for age, sex, education (see Table 3). Further inclusion of pre-stroke cognitive status and subsequently all predictor variables (stroke volume, WMH pathology, MTA pathology, left hemispheric stroke and strategic infarct) in the multivariate logistic regression resulted in persistent robust ORs (see Supplemental Table A3).

\section{Major NCD}

Major NCD after 3 months was significantly associated with stroke lesion volume $(\mathrm{OR}=1.04$ [1.02 to 1.06]) and WMH pathology $(\mathrm{OR}=3.09 \quad[1.71$ to 5.58]). The resulting ORs remained approximately constant after adjusting for age, sex, education (see Table 3). Further inclusion of pre-stroke cognitive status and subsequently all predictor variables (stroke volume, WMH pathology, 


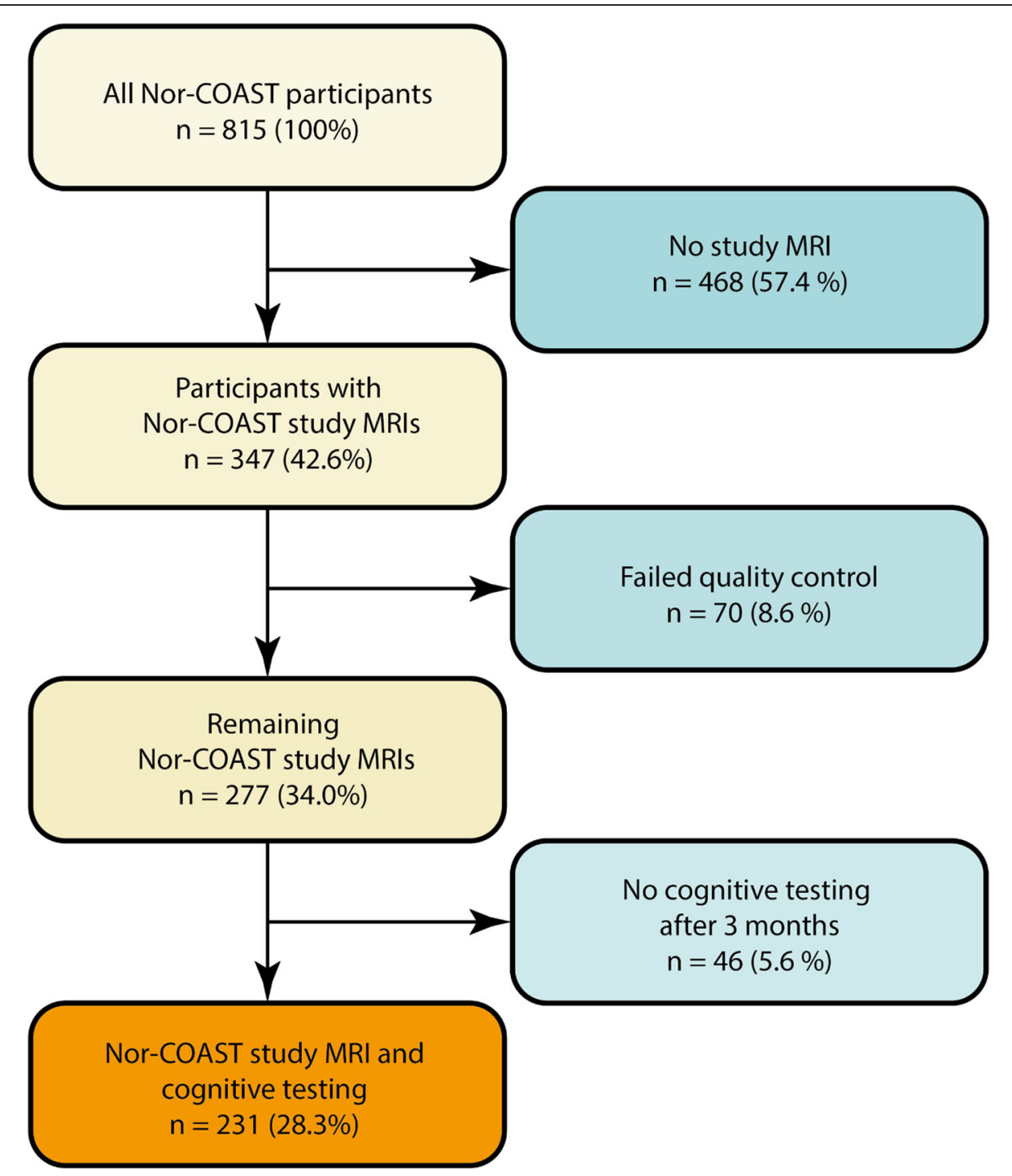

Fig. 1 Study population overview

MTA pathology, left hemispheric stroke and strategic infarct) in the multivariate logistic regression resulted in persistent robust ORs (see Supplemental Table A3).

A comparison of our findings compared to those of other studies investigating the relationship between neuroimaging biomarkers and cognitive impairment is summarized in Table 4.

\section{Discussion}

In this prospective observational cohort study of stroke survivors, we analyzed the associations between neuroimaging findings in the acute phase and NCD 3 months after the index stroke. Any NCD was significantly associated with WMH pathology, MTA pathology and left hemispheric stroke. Major NCD was significantly associated with stroke lesion volume and WMH pathology.
The fraction of patients in our study with major NCD was $28.1 \%$, which is in line with published prevalences that range from $7.4 \%$ in population-based studies to $41.3 \%$ in hospital-based studies. The wide range of prevalence is explained by varying diagnostic criteria [39].

Our results indicate that the stroke lesion volume is a significant predictor of major NCD, even though the mean stroke lesion volume is small. For every $\mathrm{ml}$ the stroke volume increases, the OR of major NCD increases by 1.04. This is consistent with another study with mainly small stroke lesion volumes (mean $7.6 \mathrm{ml}$ ) [14], where the authors reported a significant, but small proportion of the cognitive performance to be explained by the stroke size. Three studies with mean initial stroke volume above $50 \mathrm{ml}$ showed that when the initial stroke lesion volume is larger, the stroke lesion volume 
Table 1 Baseline characteristics in the cognitive outcome groups 3 months after index stroke

\begin{tabular}{|c|c|c|c|c|c|}
\hline & $\begin{array}{l}\text { Normal cognition } \\
n=103\end{array}$ & $\begin{array}{l}\text { Mild NCD } \\
n=63\end{array}$ & $\begin{array}{l}\text { Major NCD } \\
n=65\end{array}$ & $\begin{array}{l}\text { Any NCD } \\
n=128\end{array}$ & $\begin{array}{l}\text { Total } \\
n=231\end{array}$ \\
\hline Female - n (\%) & $46(44.7)$ & $23(36.5)$ & $32(49.2)$ & $55(43.0)$ & $101(43.7)$ \\
\hline Age (years) - mean (SD) & $69.4(11.2)$ & $69.7(6.7)$ & $77.5(10.3)$ & $73.6(10.7)$ & $71.8(11.1)$ \\
\hline Education (years) - mean (SD) & $13.2(3.8)$ & $12.7(3.5)$ & $10.5(3.2)$ & $11.6(3.5)$ & $12.3(3.7)$ \\
\hline Time (hrs) to admission - mean (SD) & $11(27)$ & $10(16)$ & $19(34)$ & $15(27)$ & $13(27)$ \\
\hline Hypertension - $\mathbf{n}(\%)$ & $43(41.8)$ & $31(49.2)$ & $35(53.9)$ & $66(51.6)$ & $109(47.2)$ \\
\hline Diabetes mellitus - n (\%) & $13(12.6)$ & $10(15.9)$ & $16(24.6)$ & $26(20.3)$ & $39(16.9)$ \\
\hline Hypercholesterolemia - n (\%) & $33(32.0)$ & $28(44.4)$ & $29(44.6)$ & $57(44.5)$ & $90(39.0)$ \\
\hline \multicolumn{6}{|l|}{ Smoker - n (\%) } \\
\hline previous & $36(35.0)$ & $24(38.1)$ & $30(46.2)$ & $54(42.2)$ & $90(39.0)$ \\
\hline current & $25(24.3)$ & $13(20.3)$ & $11(16.9)$ & $24(18.8)$ & $49(21.2)$ \\
\hline NIHSS ${ }^{\mathrm{a}}$ score - mean (SD) & $3.1(3.8)$ & $3.1(2.4)$ & $5.2(6.7)$ & $4.2(5.2)$ & $3.7(4.6)$ \\
\hline Previous clinical stroke - $\mathbf{n}(\%)$ & $11(10.7)$ & $7(11.1)$ & $14(21.5)$ & $21(16.4)$ & $32(13.6)$ \\
\hline$M o C A^{b}$ - mean (SD) & $26.3(2.7)$ & $24.5(3.1)$ & $19.3(5.4)^{(n=64)}$ & $22.2^{(5.0)^{(n=127)}}$ & $23.1(5.1)^{(n=230)}$ \\
\hline
\end{tabular}

${ }^{a}$ NIHSS National Institute of Health Stroke Scale. ${ }^{\mathbf{b}} M o C A$ "Montreal Cognitive Assessment Test", scored at 3 months. ${ }^{c}$ NCD Neurocognitive disorder

becomes a very strong predictor of clinical outcome measured by the modified Rankin score [40-42]. These reports are consistent with our findings. Since the odds of major NCD increases with every ml lesion volume, a small stroke lesion contributes to favorable clinical outcome after endovascular stroke therapy [43], especially when the WMH burden is low [44]. Thus, reducing the stroke lesion volume is important for reducing the odds of major NCD.

WMH pathology was in our study associated with any NCD and major NCD 3 months after stroke. This is in accordance with other studies which reported an association between WMH burden and cognitive symptoms [45-48]. The presence of severe WMH at baseline doubled the future risk of stroke and increased the risk of dementia four-fold in the "Framingham Offspring Study" [49]. Other studies confirm that WMHs are strongly associated with cerebrovascular disease [50], vascular risk factors, and dementia $[49,51,52]$. The underlying pathology is believed to reflect demyelination and axonal loss related to chronic ischemia caused by small vessel disease (SVD) [53]. SVD, for which WMH is an imaging marker, has recently been linked to glymphatic dysfunction. This glymphatic dysfunction may lead to a progression of neurodegenerative dementias in the same patients at risk for vascular dementia $[54,55]$ and it might explain the overlap between these entities.

We found a statistically significant positive association between MTA pathology and any NCD. This has been shown to varying degrees in previous studies $[9,14]$. While MTA pathology mostly occurs in the context of neurodegenerative disease [56], it has also been associated with pure vascular cognitive impairment $[57,58]$. High grades of MTA are strongly associated with dementia, especially Alzheimer's disease [56]. Fiford et al. on the other hand have suggested that vascular damage, like $\mathrm{WMH}$, is associated with greater atrophy of the hippocampus compared to controls [59]. Consequently, MTA pathology in a stroke cohort might be related to SVD as well as neurodegeneration.

Table 2 Baseline imaging findings according to cognitive outcome groups 3 months after acute stroke

\begin{tabular}{|c|c|c|c|c|c|}
\hline & $\begin{array}{l}\text { Normal cognition } \\
n=103\end{array}$ & $\begin{array}{l}\text { Mild NCD } \\
n=63\end{array}$ & $\begin{array}{l}\text { Major NCD } \\
n=65\end{array}$ & $\begin{array}{l}\text { Any NCD } \\
n=128\end{array}$ & $\begin{array}{l}\text { Total } \\
n=231\end{array}$ \\
\hline Stroke volume (ml) - mean (SD) & $7(10.8)$ & $4.1(7)$ & $16.1(27.7)$ & $10.2(21.2)$ & $8.7(17.4)$ \\
\hline Stroke volume $(\mathrm{ml})$ - median (IQR) ${ }^{\mathrm{a}}$ & $1.7(0.5-9.1)$ & $1.7(0.5-3.7)$ & $2.9(0.7-21-1)$ & $1.9(0.6-7.5)$ & $1.8(0.2-5.3)$ \\
\hline Hemorrhagic stroke - $\mathbf{n}(\%)$ & $5(4.6)$ & $7(11.1)$ & $5(7.7)$ & $12(9.4)$ & $17(7.4)$ \\
\hline Left hemisphere - $\mathrm{n}(\%)$ & $38(37.6)$ & $33(55)$ & $31(47.7)$ & $64(51.2)$ & $102(45.1)$ \\
\hline Both hemispheres - $\mathrm{n}(\%)$ & $2(2)$ & $3(5)$ & $1(1.5)$ & $4(3.2)$ & $6(2.7)$ \\
\hline Strategic infarct - n (\%) & $10(9.7)$ & $6(9.5)$ & $7(10.8)$ & $13(10.2)$ & $23(9.9)$ \\
\hline Pathologic $\mathrm{WMH}^{\mathrm{b}}$ score - $\mathrm{n}(\%)$ & $27(26.2)$ & $25(39.7)$ & $38(58.5)$ & $63(49.2)$ & $90(39)$ \\
\hline Pathologic MTA' score - $\mathbf{n}(\%)$ & $28(27.2)$ & $27(42.9)$ & $27(41.5)$ & $54(42.2)$ & $82(35.5)$ \\
\hline
\end{tabular}

${ }^{\mathrm{a}} \mathrm{QQR}$ Inter quartile range, ${ }^{\mathrm{b}} \mathrm{WMH}$ White matter hyperintensities. ${ }^{\mathrm{c}} \mathrm{MTA}$ Medial temporal lobe atrophy. ${ }^{\mathrm{d}} \mathrm{NCD}$ Neurocognitive disorder 


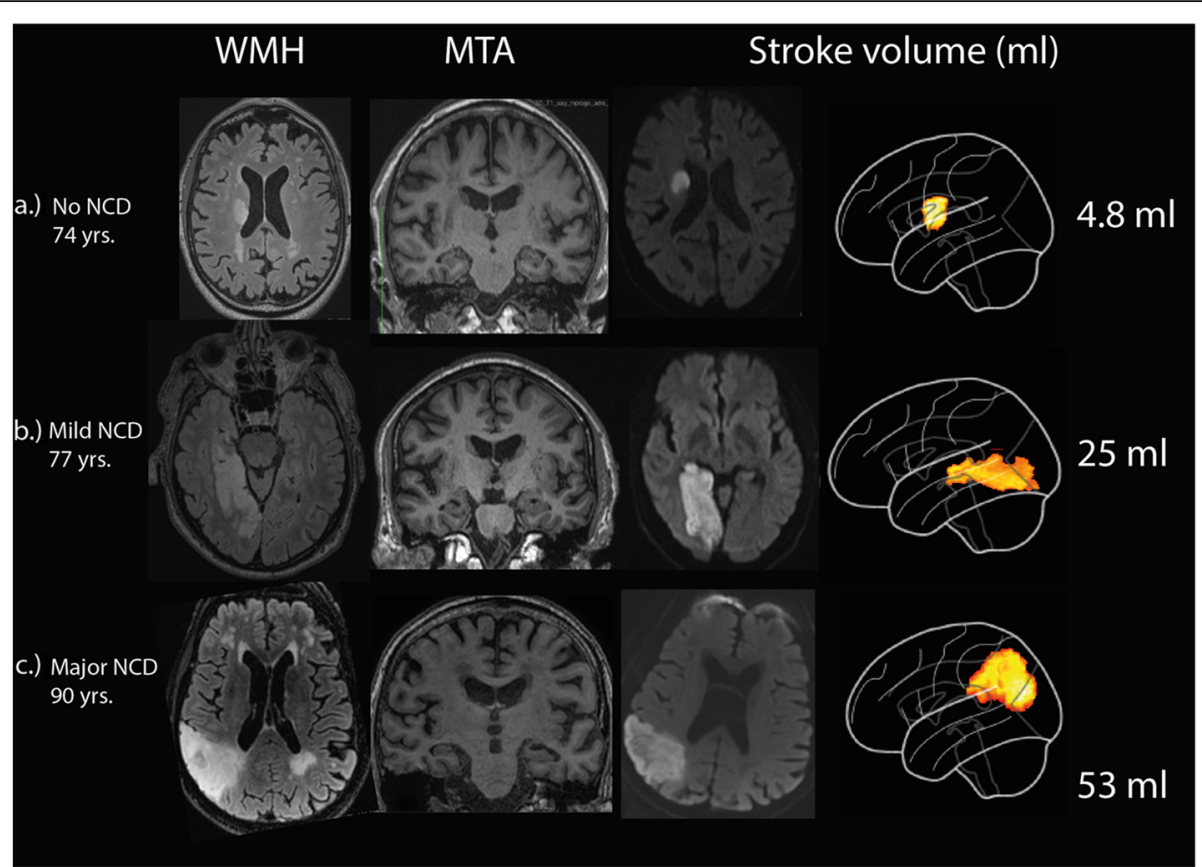

Fig. 2 Examples of WMH, MTA and stroke lesion volume for normal cognition, mild and major NCD. a.) Normal cognitive status after 3 months with normal MTA (medial temporal lobe atrophy) and WMH (white matter hyperintensity) score and an average stroke volume for this group; $\mathbf{b}$.) mild NCD (neurocognitive disorder) with a relatively small stroke volume, pathologic MTA and pathologic WMH score; c.) Major NCD with a larger stroke volume, normal MTA and pathologic WMH score

We propose that stroke lesion volume and WMH pathology are important predictors for the development of major NCD 3 months after an acute stroke. An underlying mechanism might be that an acute stroke, even with a small lesion volume, unmasks the effects of reduced brain resilience as indicated by the presence of WMHs and MTA, and triggers the development of any NCD [60]. This makes the use of imaging markers as predictors valuable and suggests that reducing stroke size may prevent the early development of post-stroke NCD. The WMH pathology and MTA pathology may be modifiable by targeting risk factors for SVD, i.e. hypertension [61, 62]. Strategic infarcts were not frequent in our study. This may explain why we did not find an association with NCD.

Although the study sample in the present study is quite representative for the general stroke population [63], a weakness of our study was that the most severely affected stroke patients were not included, which makes the results most relevant for patients with moderate to mild stroke and smaller stroke lesions.

Table 3 Results from logistic regression analyses with any or major neurocognitive disorder as outcome variable

\begin{tabular}{|c|c|c|c|c|c|c|}
\hline Imaging marker & & $\mathbf{n}$ & $\begin{array}{l}\text { Any NCD } \\
\text { OR }[95 \% \mathrm{Cl}]\end{array}$ & $p$-value & $\begin{array}{l}\text { Major NCD } \\
\text { OR }[95 \% \mathrm{Cl}]\end{array}$ & $p$-value \\
\hline \multirow[t]{2}{*}{ Stroke lesion volume (ml) } & Unadjusted & 231 & 1.01 [0.99 to 1.03] & 0.176 & $1.04[1.02$ to 1.06$]$ & 0.001 \\
\hline & Adjusted $^{a}$ & 231 & 1.01 [0.99 to 1.03$]$ & 0.275 & 1.04 [1.01 to 1.06$]$ & 0.001 \\
\hline \multirow[t]{2}{*}{ WMH pathology } & Unadjusted & 231 & 2.73 [1.56 to 4.77$]$ & 0.001 & 3.09 [1.71 to 5.58$]$ & 0.001 \\
\hline & Adjusted $^{a}$ & 231 & 2.37 [1.33 to 4.23$]$ & 0.003 & 2.54 [1.33 to 4.84$]$ & 0.005 \\
\hline \multirow[t]{2}{*}{ MTA pathology } & Unadjusted & 231 & $1.95[1.12$ to 3.41$]$ & 0.019 & 1.43 [0.79 to 2.59$]$ & 0.231 \\
\hline & Adjusted $^{a}$ & 231 & 2.13 [1.18 to 3.87$]$ & 0.013 & 1.87 [0.95 to 3.68$]$ & 0.069 \\
\hline \multirow[t]{2}{*}{ Left hemispheric stroke } & Unadjusted & 226 & 1.80 [1.05 to 3.09$]$ & 0.032 & 1.12 [0.63 to 2.01] & 0.693 \\
\hline & Adjusted $^{a}$ & 226 & $1.74[1$ to 3.04$]$ & 0.051 & 1.01 [0.53 to 1.93$]$ & 0.966 \\
\hline \multirow[t]{2}{*}{ Strategic infarct } & Unadjusted & 231 & $1.05[0.44$ to 2.51$]$ & 0.91 & 1.13 [0.44 to 2.89$]$ & 0.796 \\
\hline & Adjusted $^{a}$ & 231 & 1 [0.4 to 2.48$]$ & 1 & $1.17[0.41$ to 3.3$]$ & 0.770 \\
\hline
\end{tabular}

The Odds Ratio (OR) of any NCD or major NCD in the presence of a defined imaging marker is shown in the table above. ORs are reported unadjusted and adjusted for age, sex, and education 
Table 4 Comparison of our results with the literature

\begin{tabular}{|c|c|c|c|}
\hline Study & $\begin{array}{l}\text { Study } \\
\text { population } n\end{array}$ & $\begin{array}{l}\text { Follow-up Time } \\
\text { months }\end{array}$ & Results \\
\hline \multicolumn{4}{|c|}{ Stroke volume } \\
\hline Puy [14] & 365 & 6 & Mean (SD) stroke volume $\mathbf{7 . 6}$ (23.121) \\
\hline $\begin{array}{l}\text { Munsch } \\
{[24]}\end{array}$ & 428 & 3 & $\begin{array}{l}\text { Median }[\mathrm{IQR}] \text { stroke volume }(\mathrm{ml}) \text { for good outcome } \\
\mathbf{6}[\mathbf{0 - 1 9 6 ]} \\
\text { Median }[\mathrm{IQR}] \text { stroke volume }(\mathrm{ml}) \text { for poor outcome } \\
\mathbf{1 7}[\mathbf{0 - 2 1 1 ]}\end{array}$ \\
\hline $\begin{array}{l}\text { Jokinen } \\
\text { [9] }\end{array}$ & 560 & 36 & $\begin{array}{l}\text { Mean (SD) stroke volume (ml) } 25.7 \text { (39.8) } \\
\text { Stroke volume affected Trail Making, Stroop dots } \\
\text { and verbal fluency among others }\end{array}$ \\
\hline $\begin{array}{l}\text { Nor- } \\
\text { COAST }\end{array}$ & 231 & 3 & $\begin{array}{l}\text { Mean (SD) stroke volume }(\mathrm{ml}) \text { for mild NCD } 4.1 \text { (7) } \\
\text { Mean (SD) stroke volume }(\mathrm{ml} \text { ) for major NCD } 16.1 \\
\text { (27.7) }\end{array}$ \\
\hline
\end{tabular}

Conclusion

Increasing stroke volume is associated with lower Global Cognitive Score

Higher stroke volume is associated with lower MoCA score

Estimated total infarct volume is associated with specific cognitive deficits

Increasing stroke volume is associated with major NCD

Median [IQR] WMH score $\mathbf{1}[\mathbf{1 - 2}]$

WMH significantly predicted Trail Making time, verbal fluency and visual reproduction among others.

WMH affected Global Cognitive Score, memory score and executive function score among others

Any NCD group: $49.2 \%$ pathological WMH Major NCD group: $58.5 \%$ pathological WMH

Median [IQR] MTA 2 [0-3]

MTA is associated with processing speed, executive function, and memory

Mean (SD) MTA score for no dementia: 2.6 (1.8) Mean (SD) MTA score for dementia: 3.1 (1.9)

Normal cognition group: $27.2 \%$ pathological MTA Any NCD group: $42.2 \%$ pathological MTA

Percantage of patients with left hemispheric stroke: $47 \%$

Normal MMSE Score left hemispheric stroke: 53\% Abnormal MMSE Score lef hemispheric stroke: 47\%

Language, abstraction, and delayed recall performances were associated with left sided stroke

Percentage of left hemispheric stroke in normal post-stroke cognition group: $37.6 \%$

Percantage of left hemispheric stroke in any NCD group: $51.2 \%$

\section{Stratgic stroke}

$\begin{array}{lll}\text { Puy [14] } & 365 & 6 \\ \begin{array}{l}\text { Munsch } \\ {[24]}\end{array} & 428 & 3 \\ \text { Zhao [25] } & 410 & 3 \text { to } 6\end{array}$

Percantage of patients with strategic strokes: 25\%

Median (range) number of eloquent voxels in good outcome group: 25 (0-821) Median (range) number of eloquent voxels in poor outcome group: $138(0-13,359)$

Infarcts in left basal ganglia, left and right frontal, left parietal and left occipital influenced the MoCA score most
WMH were not associated with Global Cognitive Score

WMH are associated with specific cognitive deficits post-stroke

WMH burden was associated with poor post-stroke cognitive performance

WMH pathology is significantly associated with any and major post-stroke NCD

MTA a week determinant of Global Cognitive Score

MTA is a strong predictor of cognitive performance

MTA is the strongest predictor of memory function post-stroke

MTA is associated with any NCD

Left hemispheric stroke was moderately associated with Global Cognitive Score

Left hemispheric stroke was not associated with post-stroke cognitive outcome

Left sided stroke was associated with cognitive impairment

Left hemispheric stroke was not associated with mild or major NCD

Strategic strokes were strongly associated with Global Cognitive Score

Strategic strokes were significantly associated with poor cognitive outcome (MoCA)

Strategic infarcts were associated with MoCA score 
Table 4 Comparison of our results with the literature (Continued)

\begin{tabular}{|c|c|c|c|c|}
\hline Study & $\begin{array}{l}\text { Study } \\
\text { population } n\end{array}$ & $\begin{array}{l}\text { Follow-up Time } \\
\text { months }\end{array}$ & Results & Conclusion \\
\hline $\begin{array}{l}\text { Nor- } \\
\text { COAST }\end{array}$ & 231 & 3 & $\begin{array}{l}\text { Percantage of strategic infarcts in normal cognition } \\
\text { group: } 9.7 \% \\
\text { Percantage of strategic infarcts in mil NCD: } 9.5 \% \\
\text { Percantage of strategic infarcts in any NCD: } 10.2 \%\end{array}$ & $\begin{array}{l}\text { Strategic infarcts were not associated with } \\
\text { mild or major NCD }\end{array}$ \\
\hline
\end{tabular}

Another weakness was the relatively low number of included brain MRIs were 231, which represented $67 \%$ of the Nor-COAST study MRIs and $28 \%$ of the total NorCOAST cohort, either due to the lack of imaging or cognitive testing. This highlights the difficulty to recruit elderly patients to longitudinal neuroimaging and cognitive testing, even though the imaging protocol was significantly shortened compared to our pilot study. To increase the imaging rate, future studies should favor analyses based on standard clinical protocols or incorporate new methods for MRI sequence acceleration and reconstruction. This would also reduce a selection bias towards recruiting participants with lower NIHSS scores. A more comprehensive neuropsychological test-battery would have been preferable but was not feasible in this elderly stroke cohort. Finally short follow-up of only 3 months after stroke could perhaps have been even better. However, no significant difference has been shown between the results of 3 and 18 months post-stroke cognitive testing in Nor-COAST [64].

A strength of our study is the prospective inclusion of acute stroke patients, which were scanned with a time effective imaging protocol adapted to an older stroke cohort. This imaging protocol and the used neuroimaging markers in our study could be implemented in clinical practice.

The identified brain pathologies associated with post-stroke NCD are straightforward to assess on clinical brain-MRIs in the form of WMH-burden and MTA-score. WMH and MTA are visual scores that can be evaluated readily by neuroradiologists or experienced clinicians in a routine stroke setting. While multiple tools have been developed for the volumetric WMH-assessment, they are still almost exclusively available in a research setting. It is of importance to differentiate WMH related to healthy ageing from pathological WMH, as done in our study. Another strength is the use of a neuropsychological assessment that is based on DSM-5 and evaluated for the NorCOAST stroke cohort [8]. According to the DSM-5 criteria, impairment any cognitive domain is sufficient to diagnose NCD, and memory impairment (which is more appropriate for the diagnosis of Alzheimer's disease), is not mandatory. As such, the DSM-5 criteria help to obtain robust and reproducible cognitive assessments in stroke cohorts.

\section{Conclusions}

Development of major and any NCD 3 months after stroke is associated with acute stroke lesion volume and WMH pathology. MTA pathology is associated with any NCD. Thus, it might be possible to assess the risk of post-stroke NCD development based on clinically accessible stroke imaging. The current acute stroke therapy aims to reduce the stroke volume, and this seems to be a good strategy, not only for reducing physical disability, but also the development of major NCD in stroke survivors.

\section{Supplementary Information}

The online version contains supplementary material available at https://doi. org/10.1186/s12883-021-02117-8.

Additional file 1: Supplemental Table A1. Frequencies of pre-stroke GDS-scores in every cognitive outcome group.

Additional file 2: Supplemental Table A2. Counts of identified stroke locations.

Additional file 3: Supplemental Table A3. Results from adjusted and full logistic regression analyses with neurocognitive disorder (NCD) as dependent variable.

Additional file 4: Supplement A4. Definitions of clinical diagnoses, MTA, and Fazekas scale.

\section{Abbreviations}

NCD: Neurocognitive disorder; Nor-COAST: Norwegian Cognitive impairment After Stroke study; DSM-5: Diagnostic and Statistical Manual of Mental Disorders version 5; MRI: Magnetic resonance imaging; WMH: White matter hyperintensities; MTA: Medial temporal lobe atrophy; SD: Standard deviation; OR: Odds ratio; WHO: World Health Organization; T: Tesla; FLAIR: Fluid attenuated inversion recovery; DWl: Diffusion-weighted imaging; SWI: Susceptibility-weighted imaging; MNI: Montreal Neurological Institute; FMRIB: Oxford Centre for functional magnetic resonance imaging of the brain; FLIRT: FMRIB's Linear image registration tool - linear and intra-model registration; ITK: Insight segmentation and registration toolkit; CSV: Comma separated values; NIHSS: National Institute of Health Stroke Scale; TOAST: Trial of ORG 10172 in acute stroke treatment; GDS: Global deterioration scale; IADL: Instrumental activities of daily living; MoCA: Montreal cognitive assessment test; Cl: Confidence interval; IQR: Inter quartile range; SVD: Small vessel disease

\section{Acknowledgments}

We would also like to thank all participants and their relatives for volunteering for this project.

Thanks also to the Nor-COAST study group and staff at at St. Olavs hospital, Haukeland University Hospital, Ålesund Hospital, Vestre Viken Hospital Trust, Bærum Hospital and Oslo University Hospital, Ullevål. for providing access to scanners and images, and helping with all different practical tasks. Without you there would be no study. A special thank to Nina Sjøgren for helping with any thinkable task along the way. 


\section{Authors' contributions}

IS is the Primary Investigator of the Nor-COAST study. TS and MKB had the idea for the design of the present study and performed visual analyses of images. SA worked out the classification of cognitive status based on the DSM5 criteria. TS performed the statistical analyses with supervision by SL. TS, $M K B$, and IS were responsible for writing the present paper with additional critical input from EAa, TBW, SAa, and SL. All authors interpreted the data and read and approved the final manuscript.

\section{Funding}

This paper was generously funded by a grant from Southeastern Norway Regional Health Authority (Grant No. 2016002). The Nor-COAST study is funded by The National health association and the Norwegian University of Science and Technology (NTNU).

\section{Availability of data and materials}

The current dataset cannot be made publicly available due to Norwegian legal restrictions A portion of data can be made available upon request to interested, qualified researchers provided that an agreement is made up. The minimal data set will enable replication of the reported study findings. Requests to access the datasets should be directed to [Mona K Beyer, monbey@ous-hf.no]".

\section{Ethics approval and consent to participate}

This study has been approved by the regional committee for medical and health research, REK Nord (REK number: 2015/171 \& 2016/2306).

\section{Consent for publication}

Not applicable.

\section{Competing interests}

IS was investigator in the drug trial Boehringer-Ingelheim 1346.0023.

The other authors declare they have no competing interests.

\section{Author details}

'Division of Radiology and Nuclear Medicine, Oslo University Hospital, Oslo, Norway. ${ }^{2}$ Institute of Clinical Medicine, University of Oslo, Oslo, Norway. ${ }^{3}$ Regional Centre for Child and Youth Mental Health and Child Welfare, Department of Mental Health, Faculty of Medicine and Health Sciences, Norwegian University of Science and Technology (NTNU), Trondheim, Norway. ${ }^{4}$ Department of Neuromedicine and Movement Science, Faculty of Medicine and Health Science, Norwegian University of Science and Technology (NTNU), Trondheim, Norway. ${ }^{5}$ Department of Geriatric Medicine, Clinic of Medicine St. Olavs Hospital, Trondheim University Hospital, Trondheim, Norway. ${ }^{6}$ Department of Geriatric Medicine, Oslo University Hospital, Oslo, Norway.

Received: 11 October 2020 Accepted: 19 February 2021 Published online: 25 February 2021

\section{References}

1. Jokinen H, Melkas S, Ylikoski R, Pohjasvaara T, Kaste M, Erkinjuntti T, et al. Post-stroke cognitive impairment is common even after successful clinical recovery. Eur J Neurol. 2015;22(9):1288-94.

2. Pendlebury ST, Rothwell PM. Incidence and prevalence of dementia associated with transient ischaemic attack and stroke: analysis of the population-based Oxford vascular study. Lancet Neurol. 2019;18(3):248-58.

3. Nys GMS, van Zandvoort MJE, de Kort PLM, van der Worp HB, Jansen BPW Algra A, et al. The prognostic value of domain-specific cognitive abilities in acute first-ever stroke. Neurology. 2005;64(5):821.

4. Oksala NK, Jokinen H, Melkas S, Oksala A, Pohjasvaara T, Hietanen M, et al. Cognitive impairment predicts poststroke death in long-term follow-up. J Neurol Neurosurg Psychiatry. 2009;80(11):1230-5.

5. American Psychiatric Association, editor. Diagnostic and Statistical Manual of Mental Disorders. 5th ed: American Psychiatric Association; 2013. [cited 2020 Jun 15]. Available from: http://psychiatryonline.org/doi/book/10.1176/appi. books.9780890425596

6. Sachdev PS, Blacker D, Blazer DG, Ganguli M, Jeste DV, Paulsen JS, et al. Classifying neurocognitive disorders: the DSM-5 approach. Nat Rev Neurol. 2014;10(11):634-42.
7. Mellon L, Brewer L, Hall P, Horgan F, Williams D, Hickey A, et al. Cognitive impairment six months after ischaemic stroke: a profile from the ASPIRE-S study. BMC Neurol. 2015;15(1):31.

8. Munthe-Kaas R, Aam S, Ihle-Hansen H, Lydersen S, Knapskog A-B, Wyller TB, et al. Impact of different methods defining post-stroke neurocognitive disorder: the nor-COAST study. Alzheimers Dement. 2020;6(1):e12000.

9. Jokinen H, Koikkalainen J, Laakso HM, Melkas S, Nieminen T, Brander A, et al. Global burden of small vessel disease-related brain changes on MRI predicts cognitive and functional decline. Stroke. 2020;51(1):170-8.

10. Pendlebury ST. Dementia in patients hospitalized with stroke: rates, time course, and Clinico-pathologic factors. Int J Stroke. 2012;7(7):570-81.

11. Grysiewicz R, Gorelick PB. Key neuroanatomical structures for post-stroke cognitive impairment. Curr Neurol Neurosci Rep. 2012;12(6):703-8.

12. Schirmer MD, Donahue KL, Nardin MJ, Dalca AV, Giese A-K, Etherton MR, et al. Brain volume: an important determinant of functional outcome after acute ischemic stroke. Mayo Clin Proc. 2020;95(5):955-65.

13. Tomlinson BE, Blessed G, Roth M. Observations on the brains of demented old people. J Neurol Sci. 1970;11(3):205-42.

14. Puy L, Barbay M, Roussel M, Canaple S, Lamy C, Arnoux A, et al. Neuroimaging determinants of Poststroke cognitive performance. Stroke. 2018:49(11):2666-73.

15. Sivakumar L, Riaz P, Kate M, Jeerakathil T, Beaulieu C, Buck B, et al. White matter hyperintensity volume predicts persistent cognitive impairment in transient ischemic attack and minor stroke. Int J Stroke. 2017;12(3):264-72.

16. Molad J, Hallevi H, Korczyn AD, Kliper E, Auriel E, Bornstein NM, et al. Vascular and neurodegenerative markers for the prediction of post-stroke cognitive impairment: results from the TABASCO study. J Alzheimers Dis. 2019;70(3):889-98

17. Thijs VN, Lansberg MG, Beaulieu C, Marks MP, Moseley ME, Albers GW. Is early ischemic lesion volume on diffusion-weighted imaging an independent predictor of stroke outcome?: a multivariable analysis. Stroke. 2000;31(11):2597-602.

18. Thingstad P, Askim T, Beyer MK, Braathen $\mathrm{G}$, Ellekjær $\mathrm{H}$, Ihle-Hansen $\mathrm{H}$, et al. The Norwegian Cognitive impairment after stroke study (Nor-COAST): study protocol of a multicentre, prospective cohort study. BMC Neurol. 2018;18(1):193.

19. Hatano $\mathrm{S}$. Experience from a multicentre stroke register: a preliminary report. Bull World Health Organ. 1976;54(5):541-53.

20. Schellhorn T, Zucknick M, Askim T, Munthe-Kaas R, Ihle-Hansen H, Seljeseth YM, et al. Vascular brain pathology is more important than neurodegeneration in the pathogenesis of pre-stroke cognitive impairment. medRxiv. 2020;14:1.

21. Woolrich MW, Jbabdi S, Patenaude B, Chappell M, Makni S, Behrens T, et al. Bayesian analysis of neuroimaging data in FSL. Neurolmage. 2009; 45(Supplement 1):S173-86.

22. Jenkinson M, Smith S. A global optimisation method for robust affine registration of brain images. Med Image Anal. 2001;5(2):143-56.

23. Yushkevich PA, Piven J, Hazlett HC, Smith RG, Ho S, Gee JC, et al. Userguided 3D active contour segmentation of anatomical structures: significantly improved efficiency and reliability. Neuroimage. 2006;31(3): $1116-28$.

24. Munsch F, Sagnier S, Asselineau J, Bigourdan A, Guttmann CR, Debruxelles $\mathrm{S}$, et al. Stroke location is an independent predictor of cognitive outcome. Stroke. 2016;47(1):66-73.

25. Zhao L, Biesbroek JM, Shi L, Liu W, Kuijf HJ, Chu WW, et al. Strategic infarct location for post-stroke cognitive impairment: a multivariate lesionsymptom mapping study. J Cereb Blood Flow Metab. 2018;38(8):1299-311.

26. Lancaster JL, Woldorff MG, Parsons LM, Liotti M, Freitas CS, Rainey L, et al. Automated Talairach atlas labels for functional brain mapping. Hum Brain Mapp. 2000;10(3):120-31.

27. Notter M, Gale D, Herholz P, Markello R, Notter-Bielser M-L, Whitaker K. AtlasReader: a Python package to generate coordinate tables, region labels, and informative figures from statistical MRI images. J Open Source Softw. 2019:4(34):1257.

28. Fazekas F, Chawluk JB, Alavi A, Hurtig HI, Zimmerman RA. MR signal abnormalities at $1.5 \mathrm{~T}$ in Alzheimer's dementia and normal aging. AJR Am J Roentgenol. 1987;149(2):351-6.

29. Vernooij MW, Smits M. Structural neuroimaging in aging and Alzheimer's disease. Neuroimaging Clin N Am. 2012;22(1):33-55.

30. Scheltens $P$, Leys $D$, Barkhof F, Huglo D, Weinstein HC, Vermersch $P$, et al. Atrophy of medial temporal lobes on MRI in probable Alzheimer's disease 
and normal ageing: diagnostic value and neuropsychological correlates. J Neurol Neurosurg Psychiatry. 1992;55(10):967-72.

31. Ferreira D, Cavallin L, Larsson E-M, Muehlboeck J-S, Mecocci P, Vellas B, et al. Practical cut-offs for visual rating scales of medial temporal, frontal and posterior atrophy in Alzheimer's disease and mild cognitive impairment. J Intern Med. 2015;278(3):277-90.

32. Adams HP, Bendixen BH, Kappelle L, Biller J, Love BB, Gordon DL, et al. Classification of subtype of acute ischemic stroke. Definitions for use in a multicenter clinical trial. TOAST. Trial of org 10172 in acute stroke treatment. Stroke. 1993;24(1):35-41.

33. Reisberg B, Ferris $\mathrm{S}$, de Leon $\mathrm{M}$, Crook T. The global deterioration scale for assessment of primary degenerative dementia. Am J Psychiatr. 1982;139(9): 1136-9.

34. Nasreddine ZS, Phillips NA, Bedirian V, Charbonneau S, Whitehead V, Collin I, et al. The Montreal cognitive assessment, MoCA: a brief screening tool for mild cognitive impairment. J Am Geriatr Soc. 2005;53(4):695-9.

35. StataCorp. Stata. tata Statistical Software: Release 16. College Station: StataCorp LLC; 2019.

36. Firbank MJ, Burton EJ, Barber R, Stephens S, Kenny RA, Ballard C, et al. Medial temporal atrophy rather than white matter hyperintensities predict cognitive decline in stroke survivors. Neurobiol Aging. 2007;28(11):1664-9.

37. Dienanta S, Hamdan M, Soetjipto S, Machin A. The relevance of right and left hemisphere classification to predict cognitive outcome after stroke. J Indonesian Med Assoc. 2020 Sep 3;70(8):151-8.

38. Sagnier S, Munsch F, Bigourdan A, Debruxelles S, Poli M, Renou P, et al. The influence of stroke location on cognitive and mood impairment. A voxelbased lesion-symptom mapping study. J Stroke Cerebrovasc Dis. 2019;28(5): 1236-42.

39. Pendlebury ST, Rothwell PM. Prevalence, incidence, and factors associated with pre-stroke and post-stroke dementia: a systematic review and metaanalysis. Lancet Neurol. 2009;8(11):1006-18.

40. Amber B, Boers AM, Bot Joseph CJ, Berkhemer OA, Lingsma HF, Yoo AJ, et al. Associations of ischemic lesion volume with functional outcome in patients with acute ischemic stroke. Stroke. 2017;48(5):1233-40.

41. Gerhard V, Rico L, Ashfaq S, Armin S. Initial lesion volume is an independent predictor of clinical stroke outcome at day 90. Stroke. 2012;43(5):1266-72.

42. Zaidi SF, Amin A, Xabier U, Mouhammada J, Brian J, Maxim H, et al. Final infarct volume is a stronger predictor of outcome than recanalization in patients with proximal middle cerebral artery occlusion treated with endovascular therapy. Stroke. 2012;43(12):3238-44.

43. Elijovich L, Goyal N, Mainali S, Hoit D, Arthur AS, Whitehead M, et al. CTA collateral score predicts infarct volume and clinical outcome after endovascular therapy for acute ischemic stroke: a retrospective chart review. J Neurolntervent Surg. 2016;8(6):559-62.

44. Mistry EA, Mistry AM, Mehta T, Arora N, Starosciak AK, Rosa FDLRL, et al. White matter disease and outcomes of mechanical Thrombectomy for acute ischemic stroke. Am J Neuroradiol. 2020;41(4):639-44.

45. Brickman AM, Zahodne LB, Guzman VA, Narkhede A, Meier IB, Griffith EY, et al. Reconsidering harbingers of dementia: progression of parietal lobe white matter hyperintensities predicts Alzheimer's disease incidence. Neurobiol Aging. 2015;36(1):27-32.

46. Debette S, Markus HS. The clinical importance of white matter hyperintensities on brain magnetic resonance imaging: systematic review and meta-analysis. BMJ (Clinical research ed). 2010;341:c3666.

47. Gorelick PB, Scuteri A, Black SE, DeCarli C, Greenberg SM, ladecola C, et al. Vascular contributions to cognitive impairment and dementia. Stroke. 2011; 42(9):2672-713.

48. Haley AP, Hoth KF, Gunstad J, Paul RH, Jefferson AL, Tate DF, et al. Subjective cognitive complaints relate to white matter Hyperintensities and future cognitive decline in patients with cardiovascular disease. Am J Geriatr Psychiatry. 2009;17(11):976-85.

49. Debette S, Beiser A, DeCarli C, Au R, Himali JJ, Kelly-Hayes M, et al. Association of MRI markers of vascular brain injury with incident stroke, mild cognitive impairment, dementia, and mortality. Stroke. 2010;41(4):600-6.

50. Wardlaw JM, Smith EE, Biessels GJ, Cordonnier C, Fazekas F, Frayne R, et al. Neuroimaging standards for research into small vessel disease and its contribution to ageing and neurodegeneration. Lancet Neurol. 2013 Aug; 12(8):822-38.

51. Longstreth WT, Arnold AM, Beauchamp NJ, Manolio TA. Lefkowitz David, Jungreis Charles, et al. incidence, manifestations, and predictors of worsening white matter on serial cranial magnetic resonance imaging in the elderly. Stroke. 2005;36(1):56-61.

52. Vermeer SE, Prins ND, den Heijer T, Hofman A, Koudstaal PJ, Breteler MMB. Silent brain infarcts and the risk of dementia and cognitive decline. N Engl J Med. 2003;348(13):1215-22.

53. Prins ND, Scheltens $P$. White matter hyperintensities, cognitive impairment and dementia: an update. Nat Rev Neurol. 2015;11(3):157-65.

54. Nedergaard M, Goldman SA. Glymphatic failure as a final common pathway to dementia. Science. 2020;370(6512):50-6.

55. Mortensen KN, Sanggaard S, Mestre H, Lee H, Kostrikov S, Xavier ALR, et al. Impaired Glymphatic transport in spontaneously hypertensive rats. J Neurosci. 2019;39(32):6365-77.

56. Frisoni GB, Fox NC, Jack CR, Scheltens P, Thompson PM. The clinical use of structural MRI in Alzheimer disease. Nat Rev Neurol. 2010;6(2):67-77.

57. Kim HJ, Ye BS, Yoon CW, Noh Y, Kim GH, Cho H, et al. Cortical thickness and hippocampal shape in pure vascular mild cognitive impairment and dementia of subcortical type. Eur J Neurol. 2014:21(5):744-51.

58. O'Sullivan M, Ngo E, Viswanathan A, Jouvent E, Gschwendtner A, Saemann $P G$, et al. Hippocampal volume is an independent predictor of cognitive performance in CADASIL. Neurobiol Aging. 2009 Jun;30(6):890-7.

59. Fiford CM, Manning EN, Bartlett JW, Cash DM, Malone IB, Ridgway GR, et al. White matter hyperintensities are associated with disproportionate progressive hippocampal atrophy. Hippocampus. 2017;27(3):249-62.

60. Cumming TB, Brodtmann A. Can stroke cause neurodegenerative dementia? Int J Stroke. 2011;6(5):416-24.

61. Bath PM, Wardlaw JM. Pharmacological treatment and prevention of cerebral small vessel disease: a review of potential interventions. Int J Stroke. 2015;10(4):469-78.

62. Carole D, John C, Oguzhan C, Véronique B, Marie-Germaine B, Pierre G, et al. Effects of blood pressure lowering on cerebral white matter Hyperintensities in patients with stroke. Circulation. 2005;112(11):1644-50.

63. Kuvås KR, Saltvedt I, Aam S, Thingstad P, Ellekjær H, Askim T. $<p>$ The Risk of Selection Bias in a Clinical Multi-Center Cohort Study. Results from the Norwegian Cognitive Impairment After Stroke (Nor-COAST) Study $</ p>$ [Internet]. Vol. 12, Clinical Epidemiology. Dove Press; 2020 [cited 2021 Jan 19]. p. 1327-36. Available from: https://www.dovepress.com/the-risk-ofselection-bias-in-a-clinical-multi-center-cohort-study-res-peer-reviewedfulltext-article-CLEP

64. Aam S, Einstad MS, Munthe-Kaas R, Lydersen S, Ihle-Hansen H, Knapskog AB, et al. Post-stroke Cognitive Impairment-Impact of Follow-Up Time and Stroke Subtype on Severity and Cognitive Profile: The Nor-COAST Study. Front Neurol. 2020;11:1 [cited 2021 Jan 17]. Available from: https://www. frontiersin.org/articles/10.3389/fneur.2020.00699/full?utm_source=F-AA E\&utm_medium=EMLF\&utm_campaign=MRK_1389472_54_Neurol_2020072 8_arts_A.

\section{Publisher's Note}

Springer Nature remains neutral with regard to jurisdictional claims in published maps and institutional affiliations.

\section{Ready to submit your research? Choose BMC and benefit from:}

- fast, convenient online submission

- thorough peer review by experienced researchers in your field

- rapid publication on acceptance

- support for research data, including large and complex data types

- gold Open Access which fosters wider collaboration and increased citations

- maximum visibility for your research: over $100 \mathrm{M}$ website views per year

At BMC, research is always in progress.

Learn more biomedcentral.com/submissions 